Open Access

\title{
Longitudinal follow up of coiled intracranial aneurysms: the impact of contrast enhanced MRA in comparison to 3DTOF MRA at 3T
}

Nicoletta Anzalone*, C. De Filippis, F. Scomazzoni, G. Calori, A. ladanza, F. Simionato and C. Righi

\begin{abstract}
Background: The role of 3DTOF MRA in the follow up (FU) of coiled cerebral aneurysms is well established. Though CEMRA (Contrast Enhanced Magnetic Resonance Angiography) has demonstrated to be superior to 3DTOF MRA in showing aneurysms residual patency, its role is still debated. The aim of this study was to verify if there is an added value of CEMRA in the long term follow up of coiled treated aneurysms.

Methods: Sixty-four cerebral aneurysms treated with GDC coils regularly followed up with 3DTOF and CEMRA at 3T every year for at least four years were included in the study. Both MR exams were evaluated and scored according to Montreal scale. Residual patency rates and modifications during follow up as depicted by the two techniques on the three item score of the Montreal scale ( $\mathrm{TO}=$ total occlusion, $\mathrm{NR}=$ neck remnant and $\mathrm{AR}=$ aneurysm remnant) were registered along with management decisions. Intertechnique agreement was evaluated with respect to patency scoring in earlier and later stages of FU. Moreover the predictive value of earlier scores for both acquisitions with respect to management decision was assessed.
\end{abstract}

Results: At 1 year FU, TO to NR to AR score ratios were 31/23/10 and 22/31/11 for 3DTOF and CEMRA respectively, whereas at 4 years FU they evolved to 28/22/14 and 19/28/17 respectively. Fifteen patencies (all AR) out of 64 aneurysms were judged suitable of retreatment evaluation during FU and 8 retreatments were effectively performed after overall benefit/risk ratio considerations. All 15 reopenings were equally depicted by both techniques except one that was depicted earlier on CEMRA. Among the 9 TO at TOF MRA and NR at CEMRA at 1 year, 3 cases enlarged to NR at TOF at 4 years, most remained stable. Among the 22 cases judged NR at 1 years with both techniques, 3 cases showed enlargement at both techniques, while in other 3 cases AR was evident only at 3DCEMRA and they were not retreated.

Conclusions: CEMRA superiority in depiction of intracranial aneurysms recanalization is confirmed by our data. Nevertheless a clear impact in patient management is apparently not evident. Evidence of occlusion at 3DTOF FU may not need the addition of a CEMRA study.

\footnotetext{
* Correspondence: anzalone.nicoletta@hsr.it

Neuroradiology Department, Biostatistic Department, S Raffaele Hospital, Milan, Italy
}

\section{Biomed Central}

(c) 2016 Anzalone et al. Open Access This article is distributed under the terms of the Creative Commons Attribution 4.0 International License (http://creativecommons.org/licenses/by/4.0/), which permits unrestricted use, distribution, and reproduction in any medium, provided you give appropriate credit to the original author(s) and the source, provide a link to the Creative Commons license, and indicate if changes were made. The Creative Commons Public Domain Dedication waiver (http://creativecommons.org/publicdomain/zero/1.0/) applies to the data made available in this article, unless otherwise stated. 


\section{Background}

The role of Magnetic Resonance Angiography (MRA) in the follow up of coiled cerebral aneurysms is well recognized. Many papers have reported the high accuracy of 3DTOF MRA in depicting aneurysms recanalization when compared to DSA (Digital Subtraction Angiography) [1]. Moreover the use of higher field has shown to improve accuracy $[2,5]$.

Contrast enhanced MRA (CEMRA) has been proposed in the follow up of coiled aneurysms with the aim to reduce the impact of coils artifacts on the evaluation of aneurysm recanalization. Data from the literature are partly incoherent in showing the advantage of CEMRA in aneurysms patency depiction, partly due to the small sample size in the series $[3,4,6,7,9,10,12]$; nevertheless there is a general agreement in recognizing the advantage of contrast acquisitions in the better depiction of aneurysm remnant either small or large.

A higher sensitivity of CEMRA has been particularly proved in the evaluation of small type 1 patencies (NR), often not evident at 3DTOF where the aneurysm is judged occluded $[5,12]$. Nevertheless a clear advantage of this finding, relative to patient treatment is not known; generally such remnants are not considered to be worth a retreatment by most endovascular therapists and we do not know if they will grow to become treatable. Consequentely a clear advantage of CEMRA on a clinical perspective is unknown.

No real data on the longitudinal follow up of coiled aneurysms with the aim to compare unenhanced with enhanced acquisitions are available. Pierot L et al. [8] have reported a series of cases with coiled cerebral aneurysms followed up with 3DTOF and CEMRA with a mean interval time from treatment of 22.7 months. They came to the conclusion that 3DTOF MRA was equivalent to CEMRA in the detection of occlusion and better in showing coils. Nevertheless, their data do not cover the span of a longitudinal FU monitoring and therefore no predictiveness test is available of previous to subsequent stages of FU monitoring data. Our aim was to compare 3DTOF MRA and CEMRA in the longitudinal follow up of a series of coiled cerebral aneurysms and verify wether or not a higher depiction of neck remnants at CEMRA at earlier stages of $\mathrm{FU}$ is predictive of subsequent growth and if it played a significant impact on management decisions.

\section{Methods}

According to internal procedure all patients with ruptured or unruptured cerebral aneurysms treated with coils are followed up with MRA at 3T within one year from the procedure and then once a year for at least
5 years. DSA is performed when retreatment is considered in the presence of aneurysm recanalization.

From June 2004 to December 2010 all coiled aneurysms that were regularly followed up with 3DTOF and 3DCEMRA at $3 \mathrm{~T}$ for at least 4 years were included in this retrospective evaluation.

\section{MRA}

Magnetic resonance angiography exams were performed on 3T Philips equipment (Intera from 2004 upgraded to Achieva in December 2012, Philips, Best, The Netherlands); the parameters are shown in Table 1. 3DTOF acquisition was acquired first, followed by 3DCEMRA after bolus injection of $0.2-0.1 \mathrm{ml} / \mathrm{kg}$ of gadolinium (GDBOPTA, Bracco - Gadobutrol, Bayer Healthcare) at $2 \mathrm{ml} / \mathrm{sec}$ followed by $20 \mathrm{ml}$ of saline with a bolus track technique.

\section{Data collection}

Clinical data regarding patients (age and gender), aneurysm size, location and history (ruptured and unruptured) were collected.

Aneurysm location was classified in four groups: anterior communicating artery (AcoA), middle cerebral artery (MCA), internal carotid artery (ICA), vertebrobasilar system (VB).

Aneurysm size was classified into: small $<10 \mathrm{~mm}$ (separated in smaller and larger than $5 \mathrm{~mm}$ ), large between $10 \mathrm{~mm}$ and $25 \mathrm{~mm}$, giant $>25 \mathrm{~mm}$.

During follow up retreated cases were registered; moreover cases judged suitable for retreatment on the MRA results, but not retreated due to clinical-technical unfavourable conditions were also registered as "intention to treat" group.

\section{Data analysis}

MRA datasets included source images, MIP reconstructions and volume rendering reconstructions from both acquisitions for all the four years follow up.

Table 1 Parameters of 3DTOF and 3DCEMRA at 3T

\begin{tabular}{lcc}
\hline & 3D-TOF & CEMRA \\
\hline TE (ms) & 3.5 & 1.8 \\
TR (MS) & 23 & 5.9 \\
FOV & 250 & 220 \\
Matrix & $1024 \times 1024$ & 304 (ric. 512$)$ \\
SENSE factor & 2.5 & 3 \\
Slices & 180 & 180 \\
Voxel size & $0.5 \times 0.5 \times 1 \mathrm{~mm}$ & $0.72 \times 0.72 \times 0.80 \mathrm{~mm}$ \\
& & (ric $0.4 \times 0.4 \times 0.4)$ \\
Time acquisition & $7 \mathrm{~min}$ & $24 \mathrm{sec}$ \\
\hline
\end{tabular}


All images were evaluated by a senior neuroradiologist dedicated to MRA. 3DTOF and 3DCEMRA were evaluated blindly and separately in a random order. In all the cases the pre-treatment and end of procedure DSA exams were also evaluated to have notion of aneurysm location, shape, end procedure results and coil position. This is of particular importance in the evaluation of neck remnants.

Aneurysm status was evaluated using the three grade score on the Montreal scale [14, 15]: 0 (Total Occlusion:TO), 1 (Neck Remnant:NR), 2 (Aneurysm Remnant:AR).

Then, Montreal score was assigned to each aneurysm both by 3DTOF and CEMRA acquisitions for each year of follow up.

\section{Statistical analysis}

Kappa statistics were used to obtain intertechnique agreement at year 1 and year 4 for each investigation. The interpretation of $\mathrm{K}$ was as follow, according to Landis and Koch: $\mathrm{k}<0$ indicated no agreement; $\mathrm{k}=0-0.19$ poor agreement; $\mathrm{k}=0.20-0.39$ fair agreement, $\mathrm{k}=0.40-0.59$ moderate agreement; $\mathrm{K}=0.60-0.79$ substantial agreement; and $\mathrm{k}=0.80-1.00$ almost perfect agreement [16].

McNemar - Bowker test was calculated for each MRA technique to investigate score modifications during the entire length of follow up.

Area under receiver -operating characteristics (ROC) curves were calculated in order to compare the predictive role of 3DTOF and 3DCEMRA at 1 year on the judgement of suitableness to retreatment.

Analysis were performed with SPSS 18 version.

\section{Results}

A total of 64 cases satisfied the inclusion criteria and were included in the retrospective evaluation. The population consisted of 14 males and 50 women, age: $33-74$ years, mean $56,2+-11,1$ years, median 55 years.

Thirthy aneurysms were ruptured (46,8 \%) and 34 (53,12 \%) unruptured. Aneurysms location was ACA/ AcomA in 35 , ICA in $15, \mathrm{VB}$ in 14 . According to pretreatment aneurysms size, 50 aneurysms measured $<5 \mathrm{~mm}$, 12 between 5 and $10 \mathrm{~mm}$, and $2>10 \mathrm{~mm}$. Montreal scale score at the end of procedure was: 33 aneurysms TO, 28 NR, 3 AR.

A total of 512 MRA datasets were evaluated.

\section{Aneurysm occlusion/patency}

AT 1 year FU, TO to NR to AR Montreal scale scores ratios were 31/23/10 (48/36/16 \%) and 22/31/11 (34/49/ $17 \%)$ at 3DTOF and CEMRA respectively, whereas at 4 years FU they evolved to $28 / 22 / 14(44 / 34 / 22 \%)$ and $19 / 28 / 17$ (30/44/26 \%) respectively.

Among 22 cases scored TO in both techniques at 1 year FU, only 3 evolved to NR only at CEMRA. Among the 22 cases scored NR in both techniques at 1 year FU, 3 evolved to AR at 4 year FU in both techniques and 3 additional cases evolved to AR only at CEMRA. In 9 cases scored NR only at CEMRA, none evolved further and 3DTOF ended up scoring NR as well at 4 years FU in 3 of them (Fig. 1).

Fifteen patencies (all AR) out of 64 aneurysms (23\%) were judged suitable of retreatment evaluation during FU and $8(12,5 \%)$ retreatments were effectively performed after overall benefit/risk ratio considerations. All 15 reopenings were equally depicted by both techniques

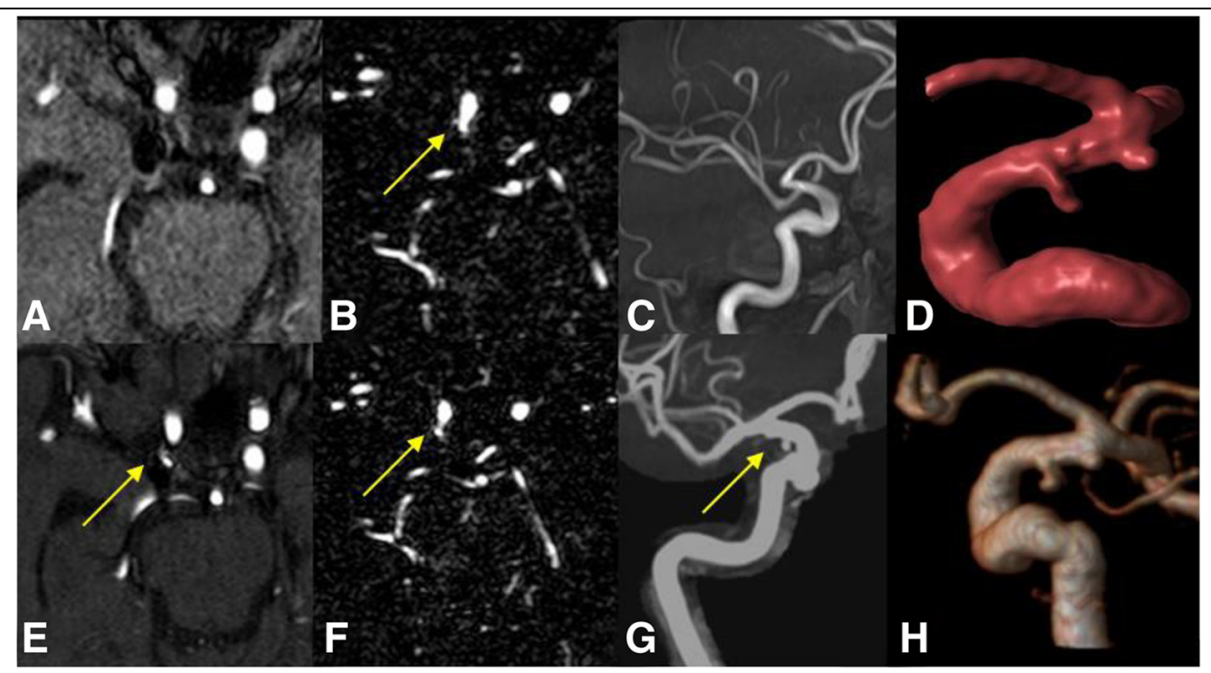

Fig. 1 Internal carotid artery treated aneurysm at 1 year (above) and 4 years (below) FU. At early follow up the remnant is not evident at 3DTOF MRA (a and $\mathbf{c}$ ) while it is evident at 3D CEMRA (b and $\mathbf{d}$, arrow)). At late follow-up the neck remnant is evident at both 3DTOF (e, $\mathbf{g}$, arrows) and 3DCEMRA (f, $\mathbf{h}$, arrow), stable 
(Fig. 2) except one that was depicted earlier on CEMRA (Fig. 3).

\section{Intermodality agreement and aneurysms grade modification during follow up according to MRA technique}

3DTOF/CEMRA intertechnique agreement in Montreal scale scoring was 0.75 (E.S.: 0,07) at 1 year and 0.72 (E.S.: 0,07 ) at 4 years $\mathrm{FU}$, showing a substantial agreement for both exams.

Both 3DTOF score $(\mathrm{p}=0.030)$ and 3DCEMRA score $(\mathrm{p}=0.011)$ significantly changed at 4 years compared to 1 year; three out of 31 and 3 out of 22 cases rated TO at 1 year FU in 3DTOF and CEMRA respectively evolved to NR at 4 years FU, while 4 out of 23 and 6 out of 31 cases rated NR at 1 year FU in 3DTOF and CEMRA respectively evolved to AR at 4 years FU (Fig. 4).

\section{Aneurysm recanalization and retreatment according to MRA techniques}

Area under ROC curves indicated that the performance of 3DTOF and 3DCEMRA in predicting retreatment was very good and very similar, being 0.94 (95\% CI: $0.88 ; 1)$ and 0.93 (95 \% CI: $0.84 ; 1)$ respectively.

\section{Discussion}

Our data confirm the higher prevalence of aneurysm recanalization together with better depiction of residual patency in favour of 3DCEMRA that has been already reported in previous studies $[5,12,13]$. Though most of the recanalizations, as known, occur within the first year from treatment, score changes according to Montreal scale are also evident in longer follow up [15]. Longitudinal evaluation of unenhanced and enhanced MRA available from our data clearly shows that this change is concordant for both techniques and, despite the higher detection of patency with 3DCEMRA, their concordance is good. Most important the data on aneurysms recanalization (AR) showed that all the retreated cases $(15,6 \%)$ were equally depicted both temporally and in score assignment by both techniques. Moreover most of AR (14 out of 17) were equally depicted at both techniques, while only 3 cases out of 17 were underscored as NR at 3DTOF; none of these 3 patencies with differential 3DTOF/CEMRA NR to AR scores was considered suitable for retreatment. Among the 15 patencies considered suitable for retreatment evaluation, only in one case 3DCEMRA anticipated the presence of aneurysm remnant that was nevertheless disclosed at 3DTOF as well, only later in time. As a consequence a clear influence on patient management of CEMRA better depiction of aneurysms patencies over 3DTOF does not emerge from our data, even if endovascular therapists may feel sometime more confident in the evaluation of aneurysm recanalization on the basis of CEMRA images. The higher detection by CEMRA of aneurysms patencies can be a sum of less sensitivity to slow flow and to susceptibility artifacts related to coils presence, artifacts known to affect 3DTOF evaluation [11]. Due to the higher sensitivity to slow flow of 3DTOF MRA, it is possible that residual flow at the neck of the treated aneurysm is not evident with this technique, as in the presence of a residual aneurysm with slow or turbolent flow where 3DTOF can underestimate the entity of the patency.

Another important result that came out from our study was the stability in time of most cases rated as NR by CEMRA, rated as TO by 3DTOF. The higher

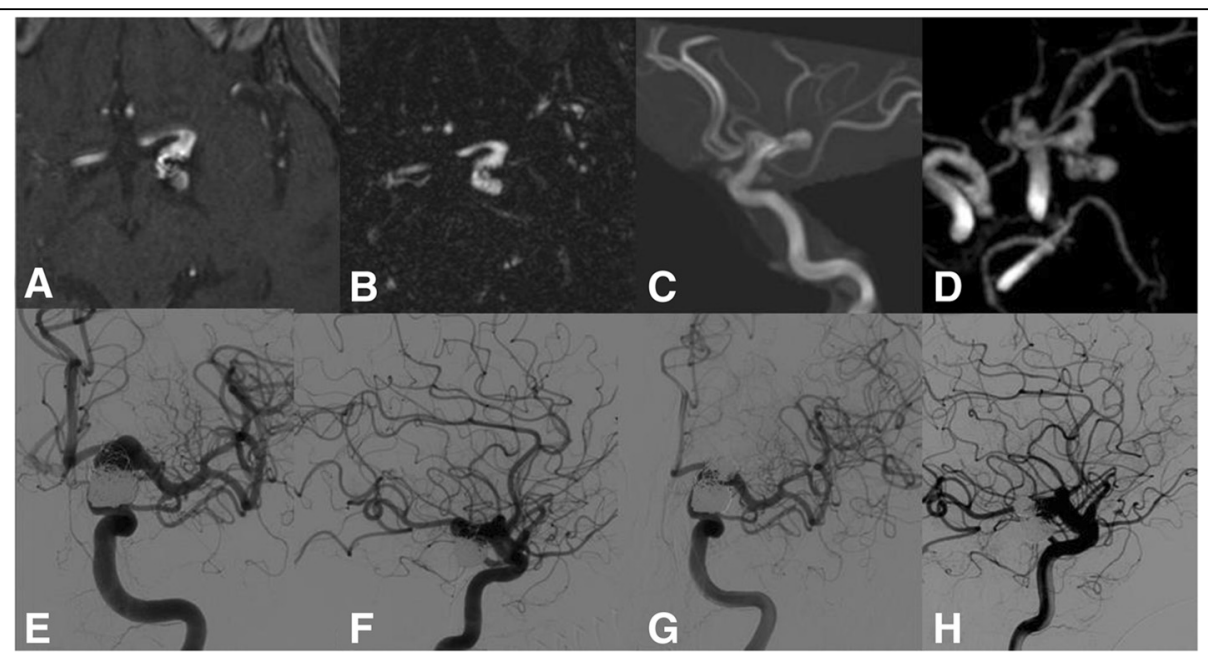

Fig. 2 Internal carotid artery retreated aneurysm. The AR was equally demonstrated at early and late follow up by both 3DTOF (a, c) and 3DCEMRA $(\mathbf{b}, \mathbf{d})$. DSA confirmed the entity of the patency $(\mathbf{e}, \mathbf{f})$ and the aneurysm was retreated with subtotal occlusion at post procedural DSA $(\mathbf{g}, \mathbf{h})$ 


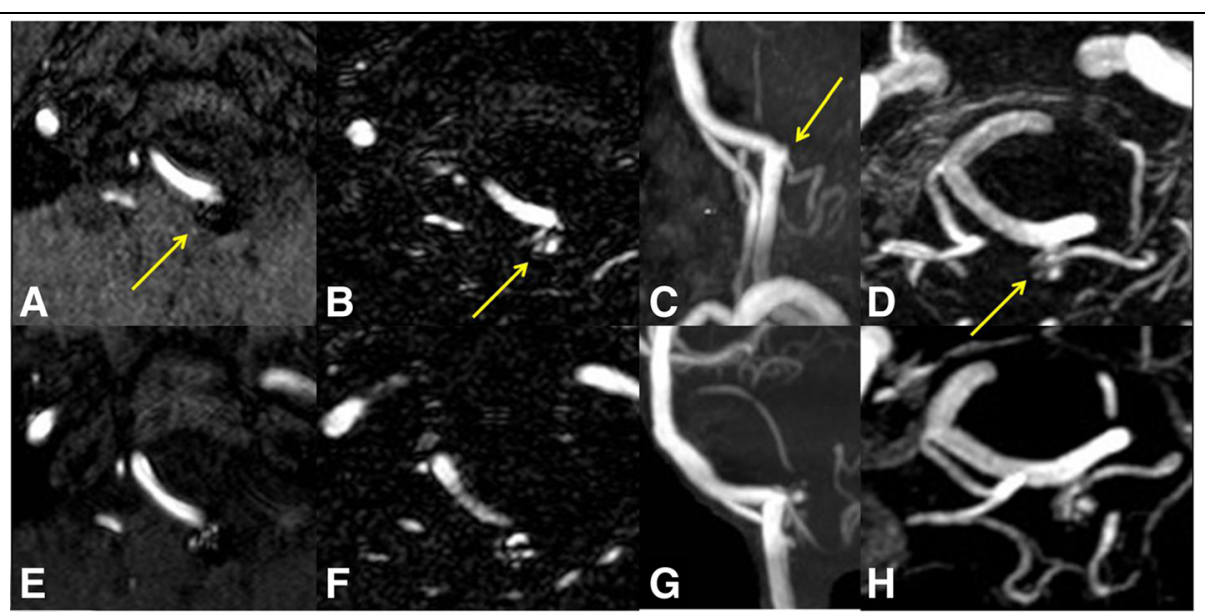

Fig. 3 Left vertebral artery treated aneurysm at 1 year (above) and 4 year (below) FU. At early follow up 3DTOF MRA (a, c, arrows) scored the aneurysm as NR, while 3DCEMRA (b, $\mathbf{d}$, arrows) as AR. At late FU the larger remnant was evident also at 3DTOF (e, $\mathbf{g})$ equally to 3DCEMRA (f, $\mathbf{h}$ )

sensitivity of 3DCEMRA in showing small remnants has been already reported but no data were previously available about the predictive role for further aneurysm recanalization. Our data showed that most of the cases scored as small patencies remained stable and no one enlarged to a higher score.

Another relevant data regard TO rate at one year that in most of the cases $(22 / 28)$ was equally depicted at both techniques and remained stable (19/22) at following FU. This observation confirms data reported in the literature on large series of treated aneurysms [15].

Sprengers et al. [7] and Pierot et al. [8] had already reported the similar performance of 3DTOF and 3DCEMRA at $3 \mathrm{~T}$ in the evaluation of aneurysm occlusion, both concluding that the latter is unnecessary in this condition. Our data show that in the presence of aneurysm occlusion at 3DTOF, 3DCEMRA can either show TO or NR, either one avoid of any tendency to further relevant growth in following FU. In this context it seems therefore possible to avoid CEMRA.
Pierot et al. [8] underlie the advantage of 3DTOF in showing coils; indeed the lesser sensitivity to susceptibility artifacts of 3DCEMRA, that allows better evidence of aneurysm enhancing remnant, obscures the images of the aneurysm shape filled of coils that can help in understanding the relationship between the coils and the remnant. On the other hand, if appropriately acquired and timed to the arterial phase, 3DCEMRA acquisitions are less disturbed by movements related artifacts than unenhanced acquisitions.

In our institution we do not regularly perform DSA to follow up coiled aneurysm and arteriography is planned only when a possible aneurysm retreatment is considered. We have a long experience in MRA follow up of coiled aneurysms and from previous published data we have reported a high diagnostic accuracy of both MRA techniques at either $1,5 \mathrm{~T}$ and 3T $[5,12]$. Moreover as well known, DSA may not always be the ideal technique to disclose aneurysm remnant, due to the frequent possible superimposition of vessels and the consequent

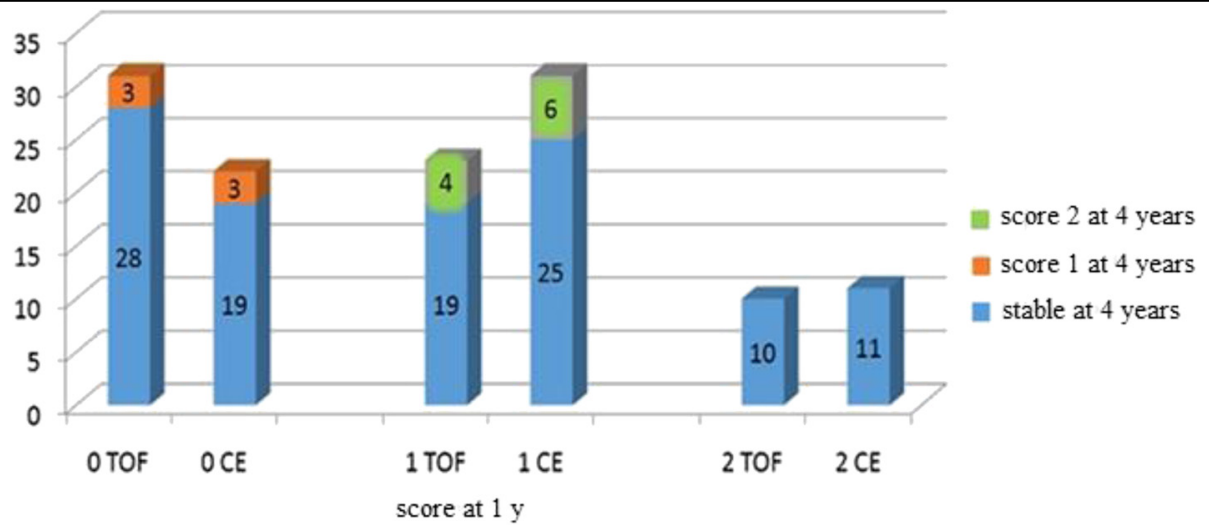

Fig. 4 Treated aneurysms status modifications at 4 years FU compared to 1 year FU 
"helmet effect" due to the presence of coils that obscure aneurysm or neck patency. We strongly support the utilization of MRA for coiled aneurysms FU evaluation considering it mature enough to substitute DSA even as gold standard for large series data reporting.

A limitation of this study is the relative small number of cases that does not allow to draw definite conclusions on the possible beneficial impact on outcome of the superiority of CEMRA in the higher demonstration of residual patency in the treated aneurysms.

Nevertheless the data of this first longitudinal follow up evaluation of 3DTOF and 3DCEMRA at 3T in coiled aneurysms show that, despite a higher prevalence of aneurysm and neck remnants demonstrated at 3DCEMRA, no modification in patients management emerged. In front of 3DTOF MRA test negative for residual patency in early FU stages, eventual patencies disclosed by CEMRA proved to be stable at later FU stages.

As opposite, in case of patencies detected at 3DTOF MRA, CEMRA may allow better depiction of the remnant and possibly disclose a larger patency.

\section{Conclusions}

Even if from our data CEMRA superiority in the depiction of intracranial aneurysms recanalization is confirmed, a clear impact in patient management is apparently not evident. As a consequence the adjunct of CEMRA to 3DTOF MRA in front of aneurysm patency may be planned on a case to case rather than on a routinary basis, following diagnostic or interventional neuroradiologists judgement. However awareness about a possible underestimation of patencies should be bared in mind.

Evidence of occlusion at 3DTOF FU may not need the addition of a CEMRA study.

\section{Competing interests}

The authors declare that they have no competing interest.

\begin{abstract}
Authors' contributions
NANA: project and protocol development, data management, manuscript editing. CDF: data collection and management. FS: data collection, manuscript writing. CG: Statistical analysis. Al: data collection and management. FS: data collection. CR: data collection, protocol design. All authors read and approved the final manuscript.
\end{abstract}

Received: 7 July 2015 Accepted: 15 October 2015

Published online: 15 December 2015

\section{References}

1. Kwee TC, Kwee RM. MR angiography in the follow-up of intracranial aneurysms treated with Guglielmi detachable coils: systematic review and meta-analysis. Neuroradiology. 2007:49:703-13.

2. Urbach $\mathrm{H}$, Dorenbeck U, von Falken hausen M, Wilhelm K, Willinek W, Schaller $C$, et al. Three dimensional time-of-flight MR angiography at 3T compared to digital subtraction angiography in the follow-up of ruptured and coiled aneurysms. Neuroradiology. 2008;50:383-9.

3. Kaufmann TJ, Huston J, Cloft HJ, Mandrekar J, Gray L, Bernstein MA, et al. A prospective trial of $3 \mathrm{~T}$ and 1.5T time-of-flight and contrast-enhanced MR angiography in the follow-up of coiled intracranial aneurysms. AJNR Am J Neuroradiol. 2010:31:912-8.

4. Pierot L, Delcourt C, Bouquigny F, Breidt D, Feuillet B, Lanoix O, et al. Follow-up of intracranial aneurysms selectively treated with coils: prospective evaluation of contrast-enhanced MR angiography. AJNR Am J Neuroradiol. 2006;27:744-9.

5. Anzalone N, Scomazzoni F, Cirillo M, Righi C, Simionato F, Cadioli M, et al. Follow-up of coiled aneurysms at 3T: comparison of 3D time-of-flight MR angiography and contrast-enhanced MR angiography. AJNR Am J Neuroradiol. 2008;29:1530-6.

6. Ramgren $B$, Siemund $\mathrm{R}$, Cronqvist $\mathrm{M}$, Undrén $\mathrm{P}$, Nilsson OG, Holtås $\mathrm{S}$, et al. Follow-up of intracranial aneurysms treated with detachable coils: comparison of 3D inflow MRA at 3T and 1.5T and contrast-enhanced MRA at 3 T with DSA. Neuroradiology. 2008;50:947-54.

7. Sprengers ME, Schaafsma JD, van Rooij WJ, van den Berg R, Rinkel GJ, Akkerman EM, et al. Evaluations of the occlusion status of coiled aneurysms with MR angiography at $3 \mathrm{~T}$ : is contrast enhancement necessary? AJNR Am J Neuroradiol. 2009;30:1665-71.

8. Pierot L, Portefaix C, Boulin A, Gauvrit JY. Follow-up of coiled intracranial aneurysms: comparison of 3D time-of-flight and contrast-enhanced magnetic resonance angiography at $3 \mathrm{~T}$ in a large, prospective series. Eur Radiol. 2012;22:2255-63.

9. Gauvrit JY, Leclerc X, Pernodet M, Lubicz B, Lejeune JP, et al. Intracranial aneurysms treated with Guglielmi detachable coils: usefulness of 6-month imaging follow-up with contrast-enhanced MR angiography. AJNR Am J Neuroradiol. 2005 Mar;26(3):515-21.

10. Cirillo M, Scomazzoni F, Cirillo L, Cadioli M, Simionato F, ladanza A, et al. Comparison of 3D TOF-MRA and 3D CE-MRA at 3T for imaging of intracranial aneurysms. Eur J Radiol. 2013 Dec;82(12):e853-9.

11. Anzalone N, Scomazzoni F, Cirillo M, Cadioli M, ladanza A, Kirchin MA, et al. Follow-up of coiled cerebral aneurysms: comparison of three-dimensional time-of-flight magnetic resonance angiography at 3 tesla with threedimensional time-of-flight magnetic resonance angiography and contrastenhanced magnetic resonance angiography at 1.5 Tesla. Invest Radiol. 2008 Aug;43(8):559-67.

12. Agid R, Willinsky RA, Lee SK, Terbrugge KG, Farb RI. Characterization of aneurysm remnants after endovascular treatment: contrast-enhanced MRA versus catheter digital subtraction angiography. AJNR Am J Neuroradiol. 2008 Sep;29(8):1570-4.

13. Raymond J, Guilbert F, Weill A, Georganos SA, Juravsky L, Lambert A, et al. Long-term angiographic recurrences after selective endovascular treatment of aneurysms with detachable coils. Stroke 2003; 34: 1398-403.

14. Raymond J, Guilbert F, Weill A, Georganos SA, Juravsky L, Lambert A, et al. Long-term angiographic recurrences after selective endovascular treatment of aneurysms with detachable coils. Stroke. 2003;34:1398-403.

15. Roy D, Milot G, Raymond J. Endovascular treatment of unruptured aneurysms. Stroke. 2001;32:1998-2004.

16. Landis JR, Koch GG. The measurement of observer agreement for categorical data. Biometrics. 1977;33:159-74.

\section{Submit your next manuscript to BioMed Central and take full advantage of:}

- Convenient online submission

- Thorough peer review

- No space constraints or color figure charges

- Immediate publication on acceptance

- Inclusion in PubMed, CAS, Scopus and Google Scholar

- Research which is freely available for redistribution 